hep-th/0006158, UTPT-00-07

\title{
Linearized Gravity about a Brane
}

\author{
Hael Collins ${ }^{\dagger}$ and Bob Holdom ${ }^{\ddagger}$ \\ Department of Physics \\ University of Toronto \\ Toronto, Ontario M5S 1A7, Canada
}

\begin{abstract}
We use the Israel condition to treat carefully the weak-field perturbations due to the presence of matter on a 3-brane embedded between two regions of antide Sitter (AdS) space with different curvature lengths. A four dimensional Newton's Law only emerges at distances that are large compared to the AdS lengths. When a scalar curvature is included in the brane action, however, it is possible to generate a four dimensional theory of gravity even when one or both of the AdS lengths is large compared to distances along the brane. In particular, we provide an example in which the AdS lengths can be larger than the millimeter experimental bound.
\end{abstract}

June, 2000

$\dagger$ haelephysics.utoronto.ca

$\ddagger$ bob.holdomQutoronto.ca 


\section{Introduction.}

One of the more intriguing new ideas in the past year has been the suggestion that the physical universe could be embedded as a hypersurface in a higher dimensional bulk space-time where the extra dimensions are non-compact. The fact that the Standard Model has been confirmed to scales approaching the electroweak scale is accounted for in these models by assuming that these fields are confined to this hypersurface, or '3-brane'. Yet since gravity describes the dynamics of space-time itself, some additional feature is needed to produce an effectively four-dimensional theory of gravity, at least on lengths greater than the millimeter scale probed. In the scenario proposed by Randall and Sundrum [1], the bulk is five dimensional anti-de Sitter $\left(\mathrm{AdS}_{5}\right)$ space-time whose curvature length provides the threshold above which the effective theory of gravity is approximately Einstein's theory in four dimensions. At distances shorter than the AdS curvature length, the extra dimension is revealed.

In studying extensions of the original Randall-Sundrum proposal, it is important to have a method for describing the effective theory of gravity seen by an inhabitant of the 3-brane. A natural approach [2] is to study the perturbations to the background metric produced when matter is placed on the brane. The problem is not as straightforward as it might seem since the presence of brane-matter can alter the position of the brane in some bulk coordinate systems. In [3] it was shown that this 'brane-bending' must be included to cancel the appearance of a spurious scalar gravity term in the effective Einstein equation in the original Randall-Sundrum scenario. A better method was recently introduced by [4], building upon their earlier work [5], in which the gauge is chosen so that the brane remains flat, even when matter is placed on the brane. As it is presented in [4], this method works best when the model has an orbifold symmetry. In this article, we shall extend the formalism to permit the study of linearized gravity in asymmetric bulk spacetime backgrounds.

Central to our discussion is a careful treatment of the boundary conditions at the brane. In the previous analyses of linearized gravity in a brane background, the energymomentum contribution from the brane to the full bulk Einstein equation was incorporated using a $\delta$-function source term. However, some of the functions that multiply this $\delta$ function become ill-defined when the bulk space-time no longer has the standard orbifold symmetry. Fortunately, the approach introduced by Israel [6] relates the discontinuities of functions evaluated on opposite sides of the brane to functions that are well-defined on the brane.

We have also considered the effect of adding a scalar curvature term to the brane action. For our purpose, we shall simply regard this curvature term as the next natural term in an effective theory expansion in powers of derivatives. Such a term can arise in some quantum field theories [7] [8] [9] from radiative corrections to gravity that involve fields confined to the brane circulating in the loops [10]. Scalar curvature terms also occur extensively in the study of the AdS/CFT correspondence where they are used to regularize the bulk action [11]. Moreover, the brane curvature term is required for the emergence of $4 d$ gravity in theories in which the brane is embedded in a flat bulk space-time [10].

The presence of a curvature term in the brane action leads to a particularly intriguing

possibility. Since an effectively $4 d$ theory of gravity from the bulk arises at distances along 
the brane greater than the AdS lengths, by adding a curvature term on the brane which has the dominant contribution to gravity at or below these scales, we can obtain a standard $4 d$ Einstein theory of gravity valid at all testable scales. With these ingredients, we can find a realistic scenario in which the AdS lengths can be above the millimeter scale and the bulk Planck scale is in the TeV range at the cost of mildly fine-tuning the coefficient of the brane curvature term.

The next section contains a detailed derivation of the weak-field perturbation to the background metric due to fields placed on the brane. Although some of this material builds on the elegant formalism of [4], we have included the derivation of the bulk equations along with the new material describing the behavior at the boundary for completeness. After a short aside on the gauge invariance of our results, in section 4 we use our results to study the effective theory of gravity along the brane for a universe consisting of a 3-brane separating two regions of $\mathrm{AdS}_{5}$ with different curvatures. In section 5 we introduce a scalar curvature term into the action and show that it can provide a source for four dimensional gravity even in theories that do not have a $4 d$ Newton's Law without it. Section 6 concludes with some comments on size of the $5 d$ Planck mass.

\section{Linearized Gravity in an Asymmetric Background.}

The action for a 3-brane embedded in a five dimensional bulk space-time can be divided into bulk and brane components. For the bulk, we use an Einstein-Hilbert action while on the brane we initially consider a minimal action containing a surface tension term plus a term for fields confined to the brane:

$$
\begin{gathered}
S=M_{5}^{3} \int d^{4} x d y \sqrt{-g}\left(2 \Lambda_{\mathrm{sgn}(y)}+R\right)+2 M_{5}^{3} \int_{\text {brane }} d^{4} x \sqrt{-h} \Delta K \\
+M_{5}^{3} \int_{\text {brane }} d^{4} x \sqrt{-h}\left(-\frac{12}{\ell}+\frac{1}{M_{5}^{3}} \mathcal{L}_{\text {fields }}\right) .
\end{gathered}
$$

$g_{a b}$ is the metric for the bulk space-time and $h_{a b}$ is the induced metric along the 3-brane. $M_{5}$ is the bulk Planck mass. We shall denote the coordinates along the brane by $x^{\mu}$, where $\mu, \nu, \cdots=0,1,2,3$; the fifth coordinate $y$ is chosen so that the brane lies at $y=0$ and $a, b, c, \ldots=0,1,2,3, y$. Notice that we have allowed the cosmological constant to have different values to either side of the brane, represented by the $\operatorname{sgn}(y)$-dependence, which leads to different metrics in the two bulk regions. When the metric changes between regions of the bulk, it is necessary to include a term in the boundary action that depends on the trace of the extrinsic curvature, $K=h^{a b} K_{a b}$, defined below. Since the boundary for the two bulk regions is provided by the brane itself, we include a term in the brane action for the difference in the extrinsic curvature for the two regions, $\left.\Delta K_{a b} \equiv K_{a b}\right|_{y=0^{-}}-\left.K_{a b}\right|_{y=0+}$. For comparison, in the standard Randall-Sundrum universe [1] $\Lambda=6 / \ell^{2}$ when the bulk metric is $d s^{2}=e^{-2|y| / \ell} \eta_{\mu \nu} d x^{\mu} d x^{\nu}+d y^{2}$ for $\mathcal{L}_{\text {fields }}=0 . \eta_{\mu \nu}=\operatorname{diag}(-1,1,1,1)$ is the four dimensional Minkowski metric.

The approach that we shall use to extract the theory of gravity for an observer on

the 3-brane is to study gravity in the weak field limit, expanding in a small perturbation 
about the classical background. The treatment of linearized gravity was first presented in [2], and then more fully in [3]. In both these papers the gauge was chosen so that the bulk metric assumed the form

$$
g_{a b}=\left(\begin{array}{cc}
e^{-2|y| / \ell} \eta_{\mu \nu}+\gamma_{\mu \nu} & 0 \\
0 & 1
\end{array}\right)
$$

where $\gamma_{\mu \nu}$ is regarded as a small perturbation. The remaining gauge freedom is used to choose $\partial^{\mu} \gamma_{\mu \nu}=0$. In these coordinates the presence of matter on the brane distorts its position in the bulk space so that it no longer lies at $y=0$. In geometries without the usual orbifold symmetry, this "brane bending" leads to subtleties in correctly imposing boundary conditions at the position of the brane. As a consequence, it is convenient to relax these gauge conditions and instead to work in a gauge where the position of the brane remains fixed at $y=0$.

The problem of finding such a gauge in order to study linearized gravity was addressed in [4]. There, the metric tensor is written using the time-slicing formalism [12] as

$$
g_{a b}=\left(\begin{array}{cc}
\hat{g}_{\mu \nu} & n_{\mu} \\
n_{\nu} & n^{2}+n_{\lambda} n^{\lambda}
\end{array}\right) \quad \quad g^{a b}=\frac{1}{n^{2}}\left(\begin{array}{cc}
\hat{g}^{\mu \nu} & -\hat{g}^{\mu \lambda} n_{\lambda} \\
-\hat{g}^{\nu \lambda} n_{\lambda} & 1
\end{array}\right)
$$

where

$$
\hat{g}_{\mu \nu}=f(y)\left(\eta_{\mu \nu}+\gamma_{\mu \nu}\right) .
$$

$n$ and $n_{\mu}$ are respectively called the lapse function and the shift vector. Here we have written a more general warp factor, $f(y)$, to allow configurations in which the brane separates two regions of $\mathrm{AdS}_{5}$ with different cosmological constants. In the limit in which the perturbations about the background are small, we consider $\gamma_{\mu \nu}, n_{\mu}$ and $\phi \equiv n^{2}-1$ all to be small quantities of roughly the same size.

The advantage of this metric is that it admits a foliation of the bulk space by hypersurfaces where $y$ is constant. The normal vector to any of these hypersurfaces can be written as

$$
N_{a}=(0,0,0,0,-n) \quad N^{a}=\frac{1}{n}\left(\hat{g}^{\mu \nu} n_{\nu},-1\right)
$$

and the induced metric along the surface is given by

$$
h_{a b}=g_{a b}-N_{a} N_{b}=\left(\begin{array}{cc}
\hat{g}_{\mu \nu} & n_{\mu} \\
n_{\nu} & n_{\lambda} n^{\lambda}
\end{array}\right) \quad h^{a b}=\frac{1}{n^{2}}\left(\begin{array}{cc}
\hat{g}^{\mu \nu}-\hat{g}^{\mu \lambda} \hat{g}^{\nu \rho} n_{\lambda} n_{\rho} & 0 \\
0 & 0
\end{array}\right)
$$

In addition to the curvature induced by this metric along a hypersurface, the bulk space also produces an extrinsic curvature structure defined by the symmetric tensor ${ }^{1}$

$$
K_{a b}=-h_{a}^{c} \nabla_{c} N_{b}
$$

1 Note that we have chosen our sign to agree with that used in [4] which is the opposite of that used in [13]. 
where the covariant derivative is with respect to the bulk metric. To leading order in the perturbations, the extrinsic curvature is then

$$
K_{\mu \nu}=\frac{1}{2 n}\left[\partial_{y} \hat{g}_{\mu \nu}-\partial_{\mu} n_{\nu}-\partial_{\nu} n_{\mu}\right]+\cdots
$$

with $K_{\mu y}=\frac{1}{2} \frac{f^{\prime}}{f} n_{\mu}$ and $K_{y y}=0$.

One of the new features in a bulk space-time without an orbifold symmetry is that some quantities become ill-defined on the brane. For example, when the cosmological constant differs on the opposite sides of the brane, it changes discontinuously at $y=0$. One solution to this problem is to allow the brane to have some finite thickness, so that the transition from one region to another proceeds smoothly. Alternatively, we can use the Israel junction equations which relate changes in bulk quantities to the brane tension and the energy-momentum tensor for fields confined to the brane. This approach avoids evaluating potentially ill-defined functions on the brane.

The behavior of gravity in the bulk is governed by the usual Einstein equation,

$$
R_{a b}-\frac{1}{2} R g_{a b}=g_{a b} \Lambda_{\mathrm{sgn}(y)}
$$

At the brane, the change in the extrinsic curvature is determined by the Israel condition [13,

$$
\Delta K_{a b}=8 \pi\left[T_{a b}-\frac{1}{3} h_{a b} T\right]
$$

$T_{a b}$ includes both the brane tension as well as fields living on the brane. Although we shall use the Israel condition to fix the behavior of the metric perturbations at the brane, it is nevertheless useful to write (2.9) in the Gauss-Codacci form by decomposing it into the transverse and orthogonal components with respect to an arbitrary $y=$ constant hypersurface,

$$
\begin{aligned}
\hat{R}+K_{\nu}^{\mu} K_{\mu}^{\nu}-K^{2} & =2 \Lambda_{\operatorname{sgn}(y)} \\
\partial_{\mu} K-\hat{\nabla}_{\nu} K_{\mu}^{\nu} & =0 \\
R_{\mu \nu} & =\frac{2}{3} \hat{g}_{\mu \nu} \Lambda_{\operatorname{sgn}(y)} .
\end{aligned}
$$

Here $\hat{R}$ is the scalar curvature associated with $\hat{g}_{\mu \nu}$ and is calculated in the appendix. Away from the brane, $y \neq 0$, the zeroth order terms from (2.11) determine the warp factor which appears in the unperturbed metric,

$$
\frac{f^{\prime 2}}{f^{2}}=-\frac{2}{3} \Lambda_{\operatorname{sgn}(y)} \quad \frac{f^{\prime \prime}}{f}=-\frac{2}{3} \Lambda_{\operatorname{sgn}(y)} .
$$

In this more general picture, $\Lambda_{\mathrm{sgn}(y)}$ can have different values for $y>0$ and $y<0$ so that $f^{\prime 2}$ becomes ill-defined on the brane.

2 Since we are writing only the equations for the bulk, we have not included the usual $\delta$-function source term for the brane in the $f^{\prime \prime}$ equation. 
The terms linear in the perturbations for the first two Gauss-Codacci equations (2.11) impose constraints on the metric:

$$
\begin{aligned}
\frac{1}{f}\left[\partial^{\mu} \partial^{\nu} \gamma_{\mu \nu}-\partial^{\lambda} \partial_{\lambda} \gamma+3 \frac{f^{\prime}}{f} \partial^{\lambda} n_{\lambda}\right] & =-3 \phi \frac{f^{\prime 2}}{f^{2}}+\frac{3}{2} \frac{f^{\prime}}{f} \partial_{y} \gamma \\
\frac{1}{f}\left[\partial^{\lambda} \partial_{\lambda} n_{\mu}-\partial_{\mu} \partial^{\nu} n_{\nu}\right] & =\frac{3}{2} \frac{f^{\prime}}{f} \partial_{\mu} \phi+\partial_{y}\left[\partial^{\nu} \gamma_{\mu \nu}-\partial_{\mu} \gamma\right]
\end{aligned}
$$

The important feature of these expressions is the appearance of the $f^{-1}$ factors on their left sides. For regions of anti-de Sitter space, $f^{-1}$ typically grows exponentially as we move farther into the bulk so that at some distance away from the brane, the assumption that $\gamma_{\mu \nu}$ and $\phi$ are small breaks down, unless we use gauge freedom to set the right sides of both expressions to zero. Therefore, in the bulk we choose, similarly to [4],

$$
\phi=\frac{1}{2} \frac{f}{f^{\prime}} \partial_{\mu} \gamma \quad \partial^{\mu} \tilde{\gamma}_{\mu \nu}=0
$$

where as $\tilde{\gamma}_{\mu \nu}$ denotes the traceless part of $\gamma_{\mu \nu}$ :

$$
\tilde{\gamma}_{\mu \nu} \equiv \gamma_{\mu \nu}-\frac{1}{4} \eta_{\mu \nu} \gamma
$$

For these equations, (2.13) constrains the shift vector to be of the following form:

$$
n_{\mu}=\frac{1}{4} \frac{f}{f^{\prime}} \partial_{\mu} \gamma+A_{\mu}
$$

Here $A_{\mu}$ is a free vector field $\left(\square A_{\mu}=0\right)$ with vanishing 4 -divergence $\left(\partial^{\mu} A_{\mu}=0\right)$. Later we shall use the gauge freedom to choose $A_{\mu}=0$.

For simplicity, we assume that all the matter is confined to the brane. On the brane, the energy-momentum tensor receives contributions from both the brane tension, which we write as $-\frac{6}{\ell}$, as well as a term $t_{\mu \nu}$ for the fields confined to the 3 -brane,

$$
T_{\mu \nu}=-\frac{3}{4 \pi} \frac{1}{\ell} \hat{g}_{\mu \nu}+t_{\mu \nu}(x) .
$$

The energy-momentum $t_{\mu \nu}$ determines the behavior of $\gamma_{\mu \nu}$ so it should also be treated as a small quantity. The surface tension of the brane is related to the discontinuity in the slope of the warp factor at the brane. Explicitly, the unperturbed piece of the Israel condition requires the usual fine-tuning of the brane tension,

$$
\left.f^{\prime}\right|_{y=0-}-\left.f^{\prime}\right|_{y=0+} \equiv \Delta\left[f^{\prime}\right]=\frac{4}{\ell} f .
$$

The behavior of $\gamma_{\mu \nu}$ in the bulk is found using the transverse components of the Einstein equation (2.11),

$$
\begin{aligned}
& \hat{R}_{\mu \nu}+\frac{1}{2} \frac{f^{\prime}}{f}\left[\partial_{\mu} n_{\nu}+\partial_{\nu} n_{\mu}+\eta_{\mu \nu} \partial^{\lambda} n_{\lambda}\right]-\frac{1}{2} f^{\prime}\left[2 \partial_{y} \gamma_{\mu \nu}+\frac{1}{2} \eta_{\mu \nu} \partial_{y} \gamma-\frac{1}{2} \eta_{\mu \nu} \partial_{y} \phi\right] \\
& -\frac{1}{2} \partial_{\mu} \partial_{\nu} \phi+\frac{1}{2}\left[f^{\prime \prime}+\frac{f^{\prime 2}}{f}\right] \eta_{\mu \nu} \phi-\frac{1}{2} f \partial_{y}^{2} \gamma_{\mu \nu}+\frac{1}{2} \partial_{y}\left[\partial_{\mu} n_{\nu}+\partial_{\nu} n_{\mu}\right]=0,
\end{aligned}
$$


where we have substituted the equations determining the warp factor (2.12). The transverse components of the Israel condition specify the boundary conditions at the brane,

$$
\Delta\left[-\frac{1}{2} f^{\prime} \phi \eta_{\mu \nu}+f \partial_{y} \gamma_{\mu \nu}-\partial_{\mu} n_{\nu}-\partial_{\nu} n_{\mu}\right]=16 \pi\left[t_{\mu \nu}-\frac{1}{3} \eta_{\mu \nu} t\right]
$$

where we have imposed the zeroth order constraint (2.18). In both these expressions we have retained only the linear terms in the metric perturbation. Both $\phi$ and $n_{\mu}$ can be eliminated from these equations through (2.14) and (2.16) so that

$$
\partial_{y}\left(f \partial_{y} \tilde{\gamma}_{\mu \nu}\right)+f^{\prime} \partial_{y} \tilde{\gamma}_{\mu \nu}+\square \tilde{\gamma}_{\mu \nu}-\frac{f^{\prime}}{f}\left[\partial_{\mu} A_{\nu}+\partial_{\nu} A_{\mu}\right]-\partial_{y}\left[\partial_{\mu} A_{\nu}+\partial_{\nu} A_{\mu}\right]=0
$$

for $y \neq 0$ and

$$
\Delta\left[f \partial_{y} \tilde{\gamma}_{\mu \nu}-\frac{1}{2} \frac{f}{f^{\prime}} \partial_{\mu} \partial_{\nu} \gamma-\partial_{\mu} A_{\nu}-\partial_{\nu} A_{\mu}\right]=16 \pi\left[t_{\mu \nu}-\frac{1}{3} \eta_{\mu \nu} t\right]
$$

at the brane, $y=0$. Finally by making a gauge transformation to eliminate $A_{\mu}$ and noting that since the metric must be continuous at the brane, so that both $f$ and $\gamma_{\mu \nu}$ are continuous there, we have the following equations for the behavior of $\gamma_{\mu \nu}$,

$$
\partial_{y}\left(f \partial_{y} \tilde{\gamma}_{\mu \nu}\right)+f^{\prime} \partial_{y} \tilde{\gamma}_{\mu \nu}+\square \tilde{\gamma}_{\mu \nu}=0
$$

in the bulk and

$$
f \Delta\left[\partial_{y} \tilde{\gamma}_{\mu \nu}\right]-\frac{1}{2} \Delta\left[\frac{f}{f^{\prime}}\right] \partial_{\mu} \partial_{\nu} \gamma=16 \pi\left[t_{\mu \nu}-\frac{1}{3} \eta_{\mu \nu} t\right]
$$

on the brane.

As in [4], we can eliminate the $\gamma$ from $(2.24)$ by taking the trace of this expression

$$
\Delta\left[\frac{f}{f^{\prime}}\right] \square \gamma=\frac{32 \pi}{3} t
$$

and inverting the operator $\square$ to write formally

$$
\left.f \partial_{y} \tilde{\gamma}_{\mu \nu}\right|_{y=0^{+}}-\left.f \partial_{y} \tilde{\gamma}_{\mu \nu}\right|_{y=0^{-}}=-16 \pi\left[t_{\mu \nu}-\frac{1}{3}\left(\eta_{\mu \nu}-\frac{\partial_{\mu} \partial_{\nu}}{\square}\right) t\right] .
$$

As for the case of the orbifold, taking the 4-divergence of this expression shows that the energy-momentum tensor for the brane fields is conserved, $\partial^{\mu} t_{\mu \nu}=0$. 


\section{Gauge Invariance.}

Before considering the weak field behavior for some specific geometries, we should show that both the bulk equation for $\tilde{\gamma}_{\mu \nu}(2.21)$ as well as the boundary condition (2.22) are left invariant under changes of coordinates that still preserve the position of the brane. Under a small change of coordinates,

$$
x^{\prime \mu}=x^{\mu}-\xi^{\mu}(x, y) \quad y^{\prime}=y-\xi^{5}(x, y)
$$

the statement that the position of the brane is left invariant is that $\xi^{5}(x, 0)=0$. The components of the metric change according to

$$
\begin{aligned}
\gamma_{\mu \nu} & =\gamma_{\mu \nu}^{\prime}-\frac{f^{\prime}}{f} \eta_{\mu \nu} \xi^{5}-\partial_{\mu} \xi_{\nu}-\partial_{\nu} \xi_{\mu} \\
n_{\mu} & =n_{\mu}^{\prime}-f \partial_{y} \xi_{\mu}-\partial_{\mu} \xi^{5} \\
\phi & =\phi^{\prime}-2 \partial_{y} \xi^{5}
\end{aligned}
$$

The trace and the traceless part of $\gamma_{\mu \nu}$ transform as

$$
\begin{aligned}
\gamma & =\gamma^{\prime}-4 \frac{f^{\prime}}{f} \xi^{5}-2 \partial^{\mu} \xi_{\mu} \\
\tilde{\gamma}_{\mu \nu} & =\tilde{\gamma}_{\mu \nu}^{\prime}-\partial_{\mu} \xi_{\nu}-\partial_{\nu} \xi_{\mu}+\frac{1}{2} \eta_{\mu \nu} \partial^{\lambda} \xi_{\lambda}
\end{aligned}
$$

If we maintain the same gauge conditions (2.14) before and after the coordinate transformation, we have further that

$$
\begin{aligned}
\partial_{y} \partial^{\lambda} \xi_{\lambda} & =-2 \xi^{5} \partial_{y}\left(\frac{f^{\prime}}{f}\right) \\
\square \xi_{\mu}+\frac{1}{2} \partial_{\mu} \partial^{\nu} \xi_{\nu} & =0 .
\end{aligned}
$$

Lastly, we note that the vector field $A_{\mu}$ transforms as follows:

$$
A_{\mu}=A_{\mu}^{\prime}-f \partial_{y} \xi_{\mu}+\frac{1}{2} \frac{f}{f^{\prime}} \partial_{\mu} \partial^{\nu} \xi_{\nu}
$$

We have used this residual gauge freedom to set $A_{\mu}=0$.

The bulk equation (2.21) is invariant provided that

$$
\left[\partial_{\mu} \partial_{\nu} \partial_{\lambda} \xi^{\lambda}+2 f^{\prime} \xi^{5} \eta_{\mu \nu}-2 \frac{f}{f^{\prime}} \partial_{\mu} \partial_{\nu} \xi^{5}\right] \partial_{y}\left(\frac{f^{\prime}}{f}\right)+f \eta_{\mu \nu} \partial_{y}\left[\xi^{5} \partial_{y}\left(\frac{f^{\prime}}{f}\right)\right]=0
$$

(2.12) implies that $f^{\prime} / f=$ constant for $y \neq 0$, so $(3.6)$ is satisfied. 
Under a coordinate transformation, we also find that the left side of the Israel condition (2.22) is invariant when

$$
\Delta\left[\partial_{\mu} \partial_{\nu} \xi_{5}\right]-2 f \eta_{\mu \nu} \Delta\left[\xi^{5} \partial_{y}\left(\frac{f^{\prime}}{f}\right)\right]=0 .
$$

These quantities are evaluated in the bulk on either side of the brane so as we have seen the second term vanishes. $\partial_{\mu} \partial_{\nu} \xi_{5}$ also vanishes since $\xi^{5}(x, y) \rightarrow 0$ as $y \rightarrow 0$, under our constraint that the position of the brane remains fixed. The gauge invariance of the Israel condition demonstrates that $\gamma(x, 0)$ is a gauge-invariant quantity determined only by the physical fields placed on the brane through (2.25). This result is important since, as we shall see in the next section, $\gamma(x, 0)$ enters the expression for the effective Einstein equations on the brane.

Before concluding this aside on the gauge invariance of (2.21)-(2.22), we should mention how the fixed-brane gauge is related to the bent-brane gauges of [2] and [3]. The appearance of the term $\Delta\left[\partial_{\mu} \partial_{\nu} \xi_{5}\right]$ in the gauge transformed Israel condition shows that if the position of the brane is not kept fixed, then gauge-dependent terms will appear in the expression for $\gamma$. Since these terms need to be subtracted from the final expression for the effective theory, it is convenient to work in a gauge in which they are absent. More importantly for a universe without an orbifold symmetry, the bending upon either side of the brane might not be equal so that extra care must be made to properly describe the boundary conditions at the brane. 3 Again, this complication is avoided when the brane's position is fixed.

\section{An Example: A Brane between Two Regions of $\mathbf{A d S}_{5}$.}

As an application, we examine in depth the behavior of a universe in which a brane with tension $-6 / \ell$ separates two regions of $\mathrm{AdS}_{5}$ with different curvatures,

$$
\Lambda=\left\{\begin{array}{ll}
6 / \ell_{1}^{2} & \text { for } y>0 \\
6 / \ell_{2}^{2} & \text { for } y<0
\end{array},\right.
$$

for which the corresponding warp factor is $f(y)=e^{-2 y / \ell_{1}}$ for $y>0$ and $f(y)=e^{2 y / \ell_{2}}$ for $y<0$. The fine-tuning condition for the brane tension $(2.18)$ is

$$
\frac{1}{\ell}=\frac{1}{2}\left[\frac{1}{\ell_{1}}+\frac{1}{\ell_{2}}\right]
$$

Since 4-derivatives appear in the equation determining $\tilde{\gamma}_{\mu \nu}$, it is convenient to Fourier transform in the directions along the brane so that in the region $y>0 \tilde{\gamma}_{\mu \nu}$ satisfies

$$
\partial_{y}\left(e^{-2 y / \ell_{1}} \partial_{y} \tilde{\gamma}_{\mu \nu}\right)-\frac{2}{\ell_{1}} e^{-2 y / \ell_{1}} \partial_{y} \tilde{\gamma}_{\mu \nu}-p^{2} \tilde{\gamma}_{\mu \nu}=0
$$

3 This point is stressed in the note accompanying equation (3.11) of [3]. 
with an analogous expression for $y<0$ involving $\ell_{2}$. For $p^{2}>0$, the solution to this differential equation is in terms of Bessel functions,

$$
\tilde{\gamma}_{\mu \nu}(p, y>0)=c_{\mu \nu}^{+}(p) e^{2 y / \ell_{1}}\left[K_{2}\left(e^{y / \ell_{1}} \ell_{1}|p|\right)+A I_{2}\left(e^{y / \ell_{1}} \ell_{1}|p|\right)\right]
$$

Since $I_{2}\left(e^{y / \ell_{1}}|p| \ell_{1}\right)$ diverges as $y \rightarrow \infty$, we set $A=0$. From (2.26) and imposing continuity of the metric across the brane, we discover the following behavior for weak fluctuations about the background solution

$$
\begin{aligned}
\tilde{\gamma}_{\mu \nu}(p, y)=\frac{16 \pi}{|p|} & {\left[t_{\mu \nu}-\frac{1}{3}\left[\eta_{\mu \nu}-\frac{p_{\mu} p_{\nu}}{p^{2}}\right] t\right] } \\
& \times \frac{e^{2 y / \ell_{1}} K_{2}\left(\ell_{2}|p|\right) K_{2}\left(\ell_{1}|p| e^{y / \ell_{1}}\right)}{K_{1}\left(\ell_{1}|p|\right) K_{2}\left(\ell_{2}|p|\right)+K_{1}\left(\ell_{2}|p|\right) K_{2}\left(\ell_{1}|p|\right)}
\end{aligned}
$$

for $y>0$ and

$$
\begin{aligned}
\tilde{\gamma}_{\mu \nu}(p, y)=\frac{16 \pi}{|p|} & {\left[t_{\mu \nu}-\frac{1}{3}\left[\eta_{\mu \nu}-\frac{p_{\mu} p_{\nu}}{p^{2}}\right] t\right] } \\
& \times \frac{e^{-2 y / \ell_{2}} K_{2}\left(\ell_{1}|p|\right) K_{2}\left(\ell_{2}|p| e^{-y / \ell_{2}}\right)}{K_{1}\left(\ell_{1}|p|\right) K_{2}\left(\ell_{2}|p|\right)+K_{1}\left(\ell_{2}|p|\right) K_{2}\left(\ell_{1}|p|\right)}
\end{aligned}
$$

for $y<0$. The value of the trace $\gamma$ at the brane is determined by the Israel condition $(2.25)$ to be

$$
\gamma(p, 0)=-\frac{32 \pi}{3 p^{2}} \frac{2}{\ell_{1}+\ell_{2}} t
$$

and along with $\tilde{\gamma}_{\mu \nu}$ is needed to find the effective theory of gravity seen by an observer confined to the brane.

A possible source for a discrepancy between a purely four dimensional theory of gravity and the effective low energy theory of gravity for an inhabitant of the 3-brane is in the appearance of deviations from the Einstein equation. The intrinsic Einstein tensor is given by [4]

$$
\hat{R}_{\mu \nu}-\frac{1}{2} \eta_{\mu \nu} \hat{R}=-\frac{1}{2} \square \tilde{\gamma}_{\mu \nu}-\frac{1}{4}\left(\partial_{\mu} \partial_{\nu} \gamma-\eta_{\mu \nu} \square \gamma\right) .
$$

As a first case, we look in the limit in which both of the lengths associated with the bulk $\mathrm{AdS}_{5}$ regions are small compared to the typical length scale probed on the brane, $\ell_{1}|p|, \ell_{2}|p| \ll 1$. Using $K_{2}(z)=2 z^{-2}+\cdots$ and $K_{1}(z)=z^{-1}+\cdots$ for small $z$, we have

$$
\tilde{\gamma}_{\mu \nu}(p, 0)=\frac{16 \pi}{p^{2}}\left[t_{\mu \nu}-\frac{1}{3}\left[\eta_{\mu \nu}-\frac{p_{\mu} p_{\nu}}{p^{2}}\right] t\right] \frac{2}{\ell_{1}+\ell_{2}}+\cdots
$$

combining this with the trace (4.7), we recover the standard theory:

$$
\hat{R}_{\mu \nu}-\frac{1}{2} \eta_{\mu \nu} \hat{R}=\frac{16 \pi}{\ell_{1}+\ell_{2}} t_{\mu \nu}+\cdots
$$


Later we shall discuss theories that deviate from this standard behavior.

We also examine whether a stationary point mass $M$ on the brane produces a $1 / r$ Newtonian potential [4]. For a point source at rest on the brane, the energy-momentum tensor has only one non-vanishing component:

$$
t_{00}(x)=\frac{M}{M_{5}^{3}} \delta^{3}(\vec{x}) \quad \text { or } \quad t_{00}(p)=2 \pi \frac{M}{M_{5}^{3}} \delta\left(p_{0}\right)
$$

where $M_{5}$ is the five dimensional Planck mass. When the velocity of a test mass is small, the gravitational field is stationary and weakly perturbed away from the background metric, we can extract the Newtonian potential by following the motion of a test mass along a geodesic. The leading piece of the geodesic equation in this Newtonian limit is

$$
\frac{d^{2} x^{i}}{d t^{2}}=\hat{\Gamma}_{00}^{i}=-\frac{1}{2} \partial_{i} \gamma_{00}=-\frac{1}{2} \partial_{i}\left(\tilde{\gamma}_{00}-\frac{1}{4} \gamma\right)
$$

from which we can extract the ordinary Newtonian potential,

$$
\frac{d^{2} x^{i}}{d t^{2}}=\partial_{i} V
$$

Typically in brane-world scenarios the standard model fields are confined to the brane by some unspecified mechanism so that a test mass is not free to move along an arbitrary geodesic, but one constrained to lie within the brane. Thus in the geodesic equation, we have used the Christoffel symbols for the induced metric.

Returning to the limit where $\ell_{1}|p|, \ell_{2}|p| \ll 1$, we note that the presence of a point mass on the brane produces a perturbation of

$$
\tilde{\gamma}_{00}(p, 0) \approx \frac{32 \pi}{3 p^{2}} \frac{2}{\ell_{1}+\ell_{2}} t_{00}
$$

while the trace $\gamma$ is fixed by (4.7). Thus when both the curvatures of the AdS regions are small compared to typical lengths probed on the brane, we recover a standard attractive $1 / r$ potential,

$$
V(r)=-\frac{1}{2} \int \frac{d^{4} p}{(2 \pi)^{4}} e^{-i p \cdot x} \gamma_{00}=-\frac{M}{M_{5}^{3}} \frac{2}{\ell_{1}+\ell_{2}} \frac{1}{r} .
$$

The preceding example represents only a slight modification of the standard RandallSundrum scenario which corresponds to setting $\ell_{1}=\ell_{2}=\ell$. However, we can also study alternative limits such as a universe in which one of the AdS lengths is small compared to the typical scales probed on the brane, $\ell_{1}|p| \ll 1$, but where the other is much larger,

4 If the test mass were allowed to move into the bulk we would have used $\Gamma_{00}^{i}$ rather than $\hat{\Gamma}_{00}^{i}$ in (4.12), which has the effect of removing the trace term, and the Newtonian potential would have been enhanced by a factor of $4 / 3$. 
$\ell_{2}|p| \gg 1$. This case can be taken as an approximation to a half-AdS, half-flat universe. In this limit, the leading contribution to the traceless piece of the metric perturbation is

$$
\tilde{\gamma}_{\mu \nu}(p, 0)=\frac{16 \pi}{|p|}\left[t_{\mu \nu}-\frac{1}{3}\left[\eta_{\mu \nu}-\frac{p_{\mu} p_{\nu}}{p^{2}}\right] t\right] \frac{1}{1+\frac{1}{2} \ell_{1}|p|-\frac{3}{2}\left(\ell_{2}|p|\right)^{-1}}+\cdots
$$

where we have used

$$
\frac{K_{1}\left(\ell_{2}|p|\right)}{K_{2}\left(\ell_{2}|p|\right)}=1-\frac{3}{2} \frac{1}{\ell_{2}|p|}+\mathcal{O}\left(\frac{1}{\ell_{2}^{2} p^{2}}\right)
$$

and neglected higher order terms as $\ell_{2}|p| \rightarrow \infty$. Since the leading behavior is $|p|^{-1}$ and not $p^{-2}$, the leading contribution to the Newtonian potential has a $1 / r^{2}$ behavior:

$$
V(r)=-\frac{8}{3 \pi} \frac{M}{M_{5}^{3}} \frac{1}{r^{2}}-\frac{4}{3} \frac{M}{M_{5}^{3}} \frac{1}{\ell_{2}} \frac{1}{r}+\frac{4}{3 \pi} \frac{M}{M_{5}^{3}} \frac{\ell_{1}^{2}}{r^{4}}+\cdots
$$

In the limit $r \ll \ell_{2}$, the second term is actually a subleading correction. This example confirms the expectation that when the brane borders a flat $5 d$ region, it is revealed by a $1 / r^{2}$ Newtonian potential.

\section{A Scalar Curvature Term in the Brane Action.}

We have used so far a minimal action on the brane consisting of only a surface tension and a lagrangian for the fields confined to the brane. Yet from an effective field theory approach to the brane action, higher order terms could be present, suppressed by powers of derivatives, that involve powers of the scalar curvature associated with the induced metric on the brane. We shall focus on the simplest such term, a scalar curvature on the brane, whose presence can produce a $4 d$ Newton's Law even in theories in which the brane borders a flat bulk region.

The presence of a scalar curvature term in the brane action, which we write with an arbitrary dimensionless coefficient $b$ by extracting a factor of the length scale associated with the brane tension,

$$
S_{\text {brane }}=M_{5}^{3} \int_{\text {brane }} d^{4} x \sqrt{-h}\left(-\frac{12}{\ell}+\frac{1}{2} b \ell \mathcal{R}+\frac{1}{M_{5}^{3}} \mathcal{L}_{\text {fields }}\right),
$$

introduces a new term in the Israel condition [13],

$$
\Delta K_{\mu \nu}=\frac{2}{\ell}+8 \pi\left[t_{\mu \nu}-\frac{1}{3} \eta_{\mu \nu} t\right]-b \frac{\ell}{2}\left[\mathcal{R}_{\mu \nu}-\frac{1}{6} h_{\mu \nu} \mathcal{R}\right] .
$$

$\mathcal{R}_{a b}$ and $\mathcal{R}$ are the Ricci tensor and curvature scalar for the induced metric $h_{a b}$. To leading

order in the perturbations, these curvatures are given by $\mathcal{R}_{\mu \nu}=\hat{R}_{\mu \nu}$ and $\mathcal{R}=\hat{R}$; their components are evaluated in the appendix. The new boundary condition imposed at $y=0$ is then

$$
f \Delta\left[\partial_{y} \tilde{\gamma}_{\mu \nu}\right]-\frac{1}{2} \Delta\left[\frac{f}{f^{\prime}}\right] \partial_{\mu} \partial_{\nu} \gamma=16 \pi\left[t_{\mu \nu}-\frac{1}{3} \eta_{\mu \nu} t\right]+\frac{1}{2} b \ell\left[\square \tilde{\gamma}_{\mu \nu}+\frac{1}{2} \partial_{\mu} \partial_{\nu} \gamma\right] .
$$


As before, we can remove the $\gamma$ terms by taking the trace of (5.3),

$$
\left(\Delta\left[\frac{f}{f^{\prime}}\right]+\frac{1}{2} b \ell\right) \square \gamma=\frac{32 \pi}{3} t
$$

which yields a condition on the traceless part of the metric perturbation similar to that in (2.26) except that an additional term with the 4-Laplacian of $\tilde{\gamma}_{\mu \nu}$ appears,

$$
\left.f \partial_{y} \tilde{\gamma}_{\mu \nu}\right|_{y=0^{+}}-\left.f \partial_{y} \tilde{\gamma}_{\mu \nu}\right|_{y=0^{-}}=\frac{1}{2} b \ell \square \tilde{\gamma}_{\mu \nu}-16 \pi\left[t_{\mu \nu}-\frac{1}{3}\left(\eta_{\mu \nu}-\frac{\partial_{\mu} \partial_{\nu}}{\square}\right) t\right] .
$$

For a universe with two regions of $\mathrm{AdS}_{5}$ separated by a 3 -brane at $y=0$, the value of the Fourier transform of the trace $\gamma$ at the brane is given by

$$
\gamma=-\frac{32 \pi}{3 p^{2}} \frac{2}{\ell_{1}+\ell_{2}+b \ell} t
$$

The solution to the bulk equation (2.23) that satisfies this new boundary condition has the form

$$
\begin{aligned}
\tilde{\gamma}_{\mu \nu}(p, y) & =\frac{16 \pi}{|p|}\left[t_{\mu \nu}-\frac{1}{3}\left[\eta_{\mu \nu}-\frac{p_{\mu} p_{\nu}}{p^{2}}\right] t\right] \\
& \times \frac{e^{2 y / \ell_{1}} K_{2}\left(\ell_{2}|p|\right) K_{2}\left(\ell_{1}|p| e^{y / \ell_{1}}\right)}{K_{1}\left(\ell_{1}|p|\right) K_{2}\left(\ell_{2}|p|\right)+K_{1}\left(\ell_{2}|p|\right) K_{2}\left(\ell_{1}|p|\right)+\frac{1}{2} b \ell|p| K_{2}\left(\ell_{1}|p|\right) K_{2}\left(\ell_{2}|p|\right)}
\end{aligned}
$$

for $y>0$ and

$$
\begin{aligned}
\tilde{\gamma}_{\mu \nu}(p, y) & =\frac{16 \pi}{|p|}\left[t_{\mu \nu}-\frac{1}{3}\left[\eta_{\mu \nu}-\frac{p_{\mu} p_{\nu}}{p^{2}}\right] t\right] \\
& \times \frac{e^{-2 y / \ell_{2}} K_{2}\left(\ell_{1}|p|\right) K_{2}\left(\ell_{2}|p| e^{-y / \ell_{2}}\right)}{K_{1}\left(\ell_{1}|p|\right) K_{2}\left(\ell_{2}|p|\right)+K_{1}\left(\ell_{2}|p|\right) K_{2}\left(\ell_{1}|p|\right)+\frac{1}{2} b \ell|p| K_{2}\left(\ell_{1}|p|\right) K_{2}\left(\ell_{2}|p|\right)}
\end{aligned}
$$

for $y<0$.

We first summarize the results that we derive below with a figure showing the appropriate effective theory of gravity seen at different scales for different relative choices for $\ell_{1}$, $\ell_{2}$ and $b \ell$. For simplicity, the lengths are assumed to be widely separated in scales in this figure so, for example, in the top plot we assume $b \ell \ll \ell_{1} \ll \ell_{2}$. The results derived in the text are more general and often allow two of these lengths to be of the same order. Unless indicated otherwise, the effective theories listed below are only valid at scales much larger or much smaller than the bounding scale $\ell_{1}, \ell_{2}$ or $b \ell$.

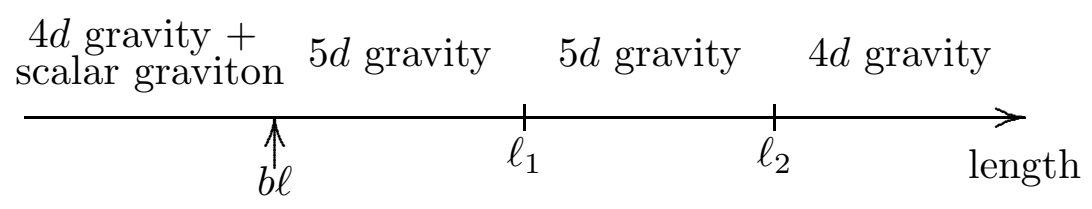




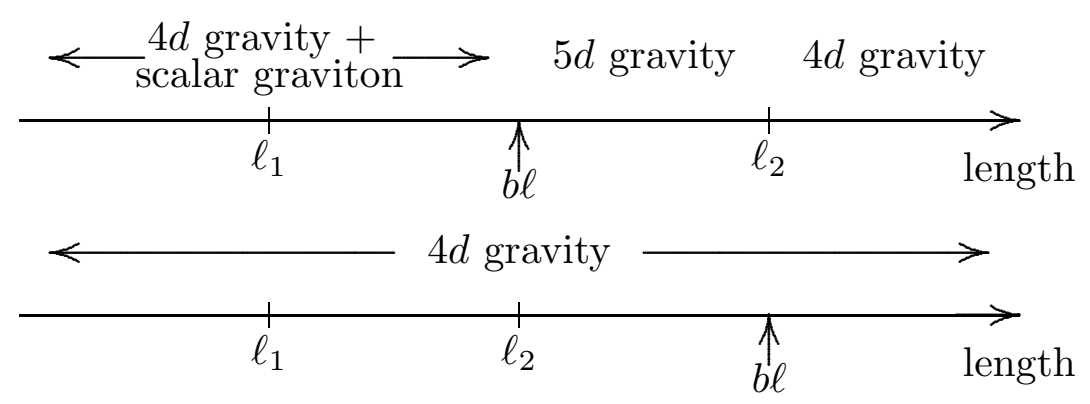

Figure 1. The effective classical theories of gravity for different regions of the $\left(\ell_{1}, \ell_{2}, b \ell\right)$ parameter space.

In the limit where both of the AdS lengths are small, $\ell_{1}|p|, \ell_{2}|p| \ll 1$, the addition of a brane scalar curvature has the familiar [13] effect of rescaling the effective Newton's constant in the low energy theory. To see this effect, we note that when we substitute the leading behavior for (5.7),

$$
\tilde{\gamma}_{\mu \nu}(p, 0)=\frac{16 \pi}{p^{2}}\left[t_{\mu \nu}-\frac{1}{3}\left[\eta_{\mu \nu}-\frac{p_{\mu} p_{\nu}}{p^{2}}\right] t\right] \frac{2}{\ell_{1}+\ell_{2}+b \ell}+\cdots,
$$

into the four dimensional Einstein tensor (4.8) and use the trace in (5.6), we obtain the correct form for four dimensional gravity,

$$
\hat{R}_{\mu \nu}-\frac{1}{2} \eta_{\mu \nu} \hat{R}=\frac{16 \pi}{\ell_{1}+\ell_{2}+b \ell} t_{\mu \nu}+\cdots .
$$

So for sufficiently large distances (the right side of Figure 1), we always recover a standard theory of gravity.

The existence of a $4 d$ curvature term in the action, however, allows us to consider new corners of the $\ell_{1}$ and $\ell_{2}$ parameter space which did not yield acceptable effective theories in the last section. If we rewrite the expression for $\tilde{\gamma}_{\mu \nu}$ on the brane as

$$
\tilde{\gamma}_{\mu \nu}(p, 0)=\frac{16 \pi}{|p|}\left[t_{\mu \nu}-\frac{1}{3}\left[\eta_{\mu \nu}-\frac{p_{\mu} p_{\nu}}{p^{2}}\right] t\right]\left[\frac{1}{2} b \ell|p|+\frac{K_{1}\left(\ell_{1}|p|\right)}{K_{2}\left(\ell_{1}|p|\right)}+\frac{K_{1}\left(\ell_{2}|p|\right)}{K_{2}\left(\ell_{2}|p|\right)}\right]^{-1}
$$

we find that since $0 \leq K_{1}(z) / K_{2}(z)<1$ for $z \geq 0$, all that is needed to recover a $1 / r$ Newtonian potential is to have $b \ell|p| \gg 1$ so that

$$
\tilde{\gamma}_{\mu \nu}(p, 0)=\frac{16 \pi}{p^{2}}\left[t_{\mu \nu}-\frac{1}{3}\left[\eta_{\mu \nu}-\frac{p_{\mu} p_{\nu}}{p^{2}}\right] t\right] \frac{2}{b \ell}+\cdots
$$

In this limit, both $\tilde{\gamma}_{\mu \nu}$ and $\gamma$ have a leading $1 / p^{2}$ behavior which automatically leads to a $1 / r$ potential:

$$
V(r)=-\frac{1}{3} \frac{M}{M_{5}^{3}} \frac{2}{b \ell}\left[\frac{4\left(\ell_{1}+\ell_{2}\right)+3 b \ell}{\ell_{1}+\ell_{2}+b \ell}\right] \frac{1}{r} .
$$

This equation is valid regardless of the scale of $\ell_{1}$ and $\ell_{2}$, compared with either the co-

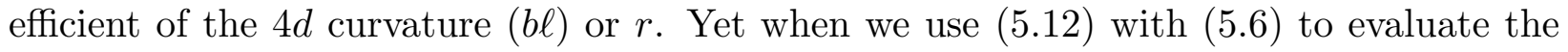


Einstein equation, we find that for arbitrary values of $\ell_{1}$ and $\ell_{2}$ that a term involving the trace of the energy-momentum tensor for the brane fields appears:

$$
\hat{R}_{\mu \nu}-\frac{1}{2} \eta_{\mu \nu} \hat{R}=\frac{16 \pi}{b \ell} t_{\mu \nu}-\frac{16 \pi}{3} \frac{1}{b \ell}\left[\frac{\ell_{1}+\ell_{2}}{b \ell+\ell_{1}+\ell_{2}}\right]\left[\eta_{\mu \nu}-\frac{\partial_{\mu} \partial_{\nu}}{\square}\right] t+\cdots
$$

If $b \ell \gg \ell_{1}, \ell_{2}$, then the extra term can be neglected and the leading behavior is that of a standard theory of four dimensional gravity,

$$
\hat{R}_{\mu \nu}-\frac{1}{2} \eta_{\mu \nu} \hat{R}=\frac{16 \pi}{b \ell} t_{\mu \nu}+\cdots
$$

in agreement with (5.10). Note we have implicitly assumed that any higher order terms in the effective theory of gravity on the brane (5.1) can be neglected.

We can also study what happens when one or both of the AdS lengths becomes infinite, while keeping $b \ell|p| \gg 1$. These limits correspond respectively to universes in which the brane is between a flat and an AdS region or is simply embedded in a flat bulk space-time. In either case, the effective theory is

$$
\hat{R}_{\mu \nu}-\frac{1}{2} \eta_{\mu \nu} \hat{R}=\frac{16 \pi}{b \ell}\left[t_{\mu \nu}-\frac{1}{3}\left(\eta_{\mu \nu}-\frac{\partial_{\mu} \partial_{\nu}}{\square}\right) t\right]+\cdots
$$

The case of a brane embedded in a flat bulk was also investigated in [10] where the effective theory on the brane was shown to contain a scalar graviton. From (5.16) it would appear that a scalar graviton is a generic feature of branes embedded in (partially) flat space-times.

Thus far we have mapped the behavior of gravity when either $\ell_{1}|p|, \ell_{2}|p| \ll 1$ or when $b \ell|p| \gg 1$ which covers most of figure 1 . What remains is the limit where $b \ell|p| \leq 1$. When $\ell_{2}|p| \gg 1 \gg \ell_{1}|p|$, then dominant contribution from (5.11) is

$$
\tilde{\gamma}_{\mu \nu}(p, 0)=\frac{16 \pi}{|p|}\left[t_{\mu \nu}-\frac{1}{3}\left[\eta_{\mu \nu}-\frac{p_{\mu} p_{\nu}}{p^{2}}\right] t\right] \frac{1}{1+\frac{1}{2}\left(b \ell+\ell_{1}\right)|p|}+\cdots
$$

while for $\ell_{2}|p|, \ell_{1}|p| \gg 1$ we have

$$
\tilde{\gamma}_{\mu \nu}(p, 0)=\frac{16 \pi}{|p|}\left[t_{\mu \nu}-\frac{1}{3}\left[\eta_{\mu \nu}-\frac{p_{\mu} p_{\nu}}{p^{2}}\right] t\right] \frac{1}{2+\frac{1}{2} b \ell|p|}+\cdots
$$

In either case, we know from our experience in the previous section, (4.16), that such a form for $\tilde{\gamma}_{\mu \nu}$ leads to a $1 / r^{2}$, or five dimensional, Newtonian potential.

\section{Conclusions.}

We have mapped out the behavior of the theory for different relative scales of the AdS lengths in the bulk, $\ell_{1}$ and $\ell_{2}$, and the coefficient of the brane curvature, $b \ell$, compared to experimentally testable lengths, represented by $1 /|p|$. When both the AdS lengths are 
both smaller than a millimeter, then regardless of the size of $b \ell$, the effective theory is standard $4 d$ gravity.

With the scalar curvature term on the brane a new regime exists, $\ell_{1}, \ell_{2} \ll b \ell$, in which a realistic classical theory of gravity also emerges. Interestingly we do not need to impose any further constraints - provided $b \ell$ is sufficiently large, $\ell_{1}$ and $\ell_{2}$ do not need to be below the millimeter limit. At distances of the order of $\ell_{1}, \ell_{2}$ or less, the brane curvature term dominates while at large distances, both the brane curvature and the effective bulk theory contribute to a standard $4 d$ theory of gravity. The largest phenomenologically acceptable $\mathrm{AdS}_{5}$ lengths depend in turn upon the largest plausible value for $b \ell$. In terms of $M_{4}$, the effective $4 d$ Planck mass, we can estimate bl using (5.15):

$$
b \ell=\frac{2 M_{4}^{2}}{M_{5}^{3}} .
$$

For example, when $M_{5}=1 \mathrm{TeV}$, then $b \ell$ is about $400 \mathrm{AU}$. Thus the theory can accommodate AdS lengths much larger than the current millimeter bound and still lie safely in the $\ell_{1}, \ell_{2} \ll b \ell$ regime.

In this regime, if $b$ is not unnaturally large, then a clear experimental signature for this universe would be the appearance of a weak correction to the Einstein equation that depends on the trace of the energy-momentum tensor (5.14). In terms of the parameterized post-Newtonian formalism [12], this extra term in the effective field equations leads to a perturbation in the parameter $\gamma$ which measures how the curvature of space depends on the presence of a rest mass. To leading order,

$$
\gamma-1=-\frac{2}{3} \frac{\ell_{1}+\ell_{2}}{b \ell}+\cdots
$$

The current value of $|\gamma-1|$ based on VLBI observations is less than $3 \times 10^{-4}$ [14]. Thus for a symmetric universe in which $\ell_{1}=\ell_{2}=\ell$, this limit constrains $b \geq 4000$. Alternatively if $M_{5} \approx 1 \mathrm{TeV}$, then from (6.1) the model can accommodate AdS lengths as large as $10^{10} \mathrm{~m}$.

We have here extended the approach of using a gauge in which the position of the brane remains fixed [4] to allow an unambiguous statement of the boundary conditions in a setting more general than the standard orbifold universes. A correct understanding of these boundary conditions is crucial for determining the form of the effective theory of gravity on the brane. Our results can be readily generalized. For example, the brane itself might be given some non-trivial global curvature as in [15] and 13] where the AdS bulk metric is replaced with an AdS-Schwarzschild metric. It is also straightforward to include more general actions on the brane; their existence would simply add extra terms to the right side of the Israel equation, (2.10).

\section{Appendix A. Conventions and Components.}

The conventions that we have used throughout use a metric with signature $(-,+, \cdots,+)$ and a Riemann curvature tensor defined by

$$
-R_{b c d}^{a} \equiv \partial_{d} \Gamma_{b c}^{a}-\partial_{c} \Gamma_{b d}^{a}+\Gamma_{e d}^{a} \Gamma_{b c}^{e}-\Gamma_{e c}^{a} \Gamma_{b d}^{e} .
$$


$\mathcal{R}_{b c d}^{a}$ and $\hat{R}_{\mu \nu \lambda}^{\rho}$, the curvature tensors corresponding respectively to the induced metric $h_{a b}$ or its transverse components $\hat{g}_{\mu \nu}$, use the same sign convention.

In extracting the terms in the Einstein equation and the Israel condition, which are linear in the perturbation about the background metric, we have used the following expressions for the Christoffel symbols for the induced metric,

$$
\hat{\Gamma}_{\mu \nu}^{\lambda}=\frac{1}{2}\left[\partial_{\mu} \gamma_{\nu}^{\lambda}+\partial_{\nu} \gamma_{\nu}^{\lambda}-\partial^{\lambda} \gamma_{\mu \nu}\right]
$$

while for the bulk metric we have

$$
\begin{aligned}
& \Gamma_{\mu \nu}^{\lambda}=\hat{\Gamma}_{\mu \nu}^{\lambda}+\frac{1}{2} \frac{f^{\prime}}{f} n^{\lambda} \eta_{\mu \nu} \\
& \Gamma_{\mu \nu}^{y}=-\frac{1}{2} \frac{1}{n^{2}} f^{\prime} \eta_{\mu \nu}-\frac{1}{2}\left[f^{\prime} \gamma_{\mu \nu}+f \partial_{y} \gamma_{\mu \nu}\right]+\frac{1}{2}\left(\partial_{\mu} n_{\nu}+\partial_{\nu} n_{\mu}\right) \\
& \Gamma_{\mu y}^{\lambda}=\frac{1}{2} \frac{f^{\prime}}{f} \eta_{\mu}^{\lambda}+\frac{1}{2} \partial_{y} \gamma_{\mu}^{\lambda}+\frac{1}{2} \frac{1}{f}\left(\partial_{\mu} n^{\lambda}-\partial^{\lambda} n_{\mu}\right) \\
& \Gamma_{\mu y}^{y}=-\frac{1}{2} \frac{f^{\prime}}{f} n_{\mu}+\frac{1}{2} \partial_{\mu} \phi \\
& \Gamma_{y y}^{\lambda}=\frac{1}{2} \frac{1}{f}\left[2 \partial_{y} n^{\lambda}-\partial^{\lambda} \phi\right] \\
& \Gamma_{y y}^{y}=\frac{1}{2} \partial_{y} \phi .
\end{aligned}
$$

The linear contributions to the transverse components of the Ricci tensor for the bulk and the induced metrics are respectively

$$
\begin{aligned}
R_{\mu \nu}= & \hat{R}_{\mu \nu}+\frac{1}{2}\left[f^{\prime \prime}+\frac{f^{\prime 2}}{f}\right]\left[\frac{1}{n^{2}} \eta_{\mu \nu}+\gamma_{\mu \nu}\right]+\frac{1}{2} \partial_{\mu} \partial_{\nu} \phi+\frac{1}{2} f \partial_{y}^{2} \gamma_{\mu \nu} \\
& +\frac{1}{2} f^{\prime}\left[2 \partial_{y} \gamma_{\mu \nu}+\frac{1}{2} \eta_{\mu \nu} \partial_{y} \gamma-\frac{1}{2} \eta_{\mu \nu} \partial_{y} \phi\right] \\
& -\frac{1}{2} \frac{f^{\prime}}{f}\left[\partial_{\mu} n_{\nu}+\partial_{\nu} n_{\mu}+\eta_{\mu \nu} \partial^{\lambda} n_{\lambda}\right]-\frac{1}{2} \partial_{y}\left(\partial_{\mu} n_{\nu}+\partial_{\nu} n_{\mu}\right) \\
\hat{R}_{\mu \nu}= & \frac{1}{2}\left[\partial_{\mu} \partial_{\nu} \gamma+\partial^{\lambda} \partial_{\lambda} \gamma_{\mu \nu}-\partial_{\mu} \partial^{\lambda} \gamma_{\lambda \nu}-\partial_{\nu} \partial^{\lambda} \gamma_{\mu \lambda}\right] .
\end{aligned}
$$

Finally, the linear terms in the extrinsic curvature are

$$
K_{\nu}^{\mu}=\frac{1}{2 n}\left[\frac{f^{\prime}}{f} \eta_{\nu}^{\mu}+\partial_{y} \gamma_{\nu}^{\mu}-\frac{1}{f}\left(\partial^{\mu} n_{\nu}+\partial_{\nu} n^{\mu}\right)\right]
$$

where $f(y)$ is the appropriate warp factor for either bulk region. 


\section{References}

[1] L. Randall and R. Sundrum, "An alternative to compactification," Phys. Rev. Lett. 83, 4690 (1999) hep-th/9906064.

[2] J. Garriga and T. Tanaka, "Gravity in the brane-world," hep-th/9911055.

[3] S. B. Giddings, E. Katz and L. Randall, "Linearized gravity in brane backgrounds," JHEP 0003, 023 (2000) hep-th/0002091.

[4] I. Y. Aref'eva, M. G. Ivanov, W. Muck, K. S. Viswanathan and I. V. Volovich, "Consistent linearized gravity in brane backgrounds," hep-th/0004114.

[5] M. G. Ivanov and I. V. Volovich, "Metric fluctuations in brane worlds," hepth/9912242 and W. Muck, K. S. Viswanathan and I. V. Volovich, "Geodesics and Newton's law in brane backgrounds," hep-th/0002132.

[6] W. Israel, "Singular Hypersurfaces And Thin Shells In General Relativity," Nuovo Cim. B44S10, 1 (1966).

[7] D. M. Capper, "On Quantum Corrections To The Graviton Propagator," Nuovo Cim. A25, 29 (1975).

[8] S. L. Adler, Phys. Rev. Lett. 44, 1567 (1980); S. L. Adler, Phys. Lett. B95, 241 (1980);

S. L. Adler, Rev. Mod. Phys. 54, 729 (1982); Erratum-ibid. 55, 837 (1983).

[9] A. Zee, "Calculating Newton's Gravitational Constant In Infrared Stable Yang-Mills Theories," Phys. Rev. Lett. 48, 295 (1982).

[10] G. Dvali, G. Gabadadze and M. Porrati, "4D gravity on a brane in 5D Minkowski space," hep-th/0005016.

[11] See for example R. Emparan, C. V. Johnson and R. C. Myers, Phys. Rev. D60, 104001 (1999) hep-th/9903238 and V. Balasubramanian and P. Kraus, Commun. Math. Phys. 208, 413 (1999) [hep-th/9902121].

[12] C. W. Misner, K. S. Thorne and J. A. Wheeler, Gravitation, (W. H. Freeman: San Francisco) 1973.

[13] H. Collins and B. Holdom, "Brane cosmologies without orbifolds," hep-ph/0003173.

[14] C. M. Will, Theory and Experiment in Gravitational Physics, Cambridge University Press: Cambridge (1993) and C. M. Will, "The confrontation between general relativity and experiment: A 1998 update," gr-qc/9811036.

[15] P. Kraus, "Dynamics of anti-de Sitter domain walls," JHEP 9912, 011 (1999) hepth/9910149]. 